\title{
Perovskite-type oxides as susceptor materials in dielectric heating
}

\author{
Y. Zhang-Steenwinkel $\cdot$ H. L. Castricum • \\ A. Bliek · E. Esveld
}

Received: 3 January 2004 / Accepted: 18 August 2004 / Published online: 24 May 2007

(C) Springer Science+Business Media, LLC 2007

\begin{abstract}
Dielectric properties of $\mathrm{La}_{1-x} \mathrm{Ce}_{x} \mathrm{MnO}_{3}$ perovskites are investigated in order to assess the heating behaviour in the wider context of the use of perovskite coatings in microwave assisted soot filter regeneration. Dielectric permittivities in the radio and microwave region for these perovskites were determined at room temperature. The dielectric constant and dielectric loss are related to ionic conduction at low frequencies, while at microwave frequencies storage and loss mainly proceed through reorientation of molecular dipoles. The dielectric constant rises for a higher degree of $\mathrm{La}$ substitution by $\mathrm{Ce}$, which is explained by an increase of the number of cation/oxygen vacancies. Concurrently the mean perovskite crystallite size decreases, which is possibly related to defect formation. The dielectric constant declines for $x \geq 0.3$, along with the formation of a separate, low dielectric permittivity $\mathrm{CeO}_{2}$ phase. The La-Ce-Mn perovskites are further shown to exhibit a high thermal stability during repeated heating/cooling cycles.
\end{abstract}

\section{Introduction}

Perovskite-type oxides of the general formula $A_{1-x} A_{x}{ }^{\prime}$ $\mathrm{B}_{1-y} \mathrm{~B}_{y}{ }^{\prime} \mathrm{O}_{3}$ are widely studied dielectric materials [1-4].

Y. Zhang-Steenwinkel · H. L. Castricum ·

A. Bliek $(\bowtie)$

Department of Chemical Engineering, University

of Amsterdam, Nieuwe Achtergracht 166, 1018 WV

Amsterdam, The Netherlands

e-mail: bliek@science.uva.nl

E. Esveld

A \& F, Wagneningen University, P.O. Box 17,

6700 AA Wageningen, The Netherlands
The combination of very low losses, rather high dielectric constant and high thermal stability at ultra high frequencies, renders these materials suitable for use as microwave resonators and filters [5, 6]. Typical examples are $\operatorname{Sr}\left(\mathrm{Zn}_{1 / 3} \mathrm{Nb}_{2 / 3}\right) \mathrm{O}_{3}-\mathrm{Ba}\left(\mathrm{Zn}_{1 / 3} \mathrm{Nb}_{2 / 3}\right) \mathrm{O}_{3}$ and $\mathrm{Ba}\left(\mathrm{Y}_{1 / 2} \mathrm{Ta}_{1 / 2}\right) \mathrm{O}_{3}$ [7-9].

Perovskite-type oxides have also been widely used as catalysts in various oxidative and reductive reactions [10]. Some perovskites may even be considered as an alternative to expensive noble metals (e.g., $\mathrm{Pd}$ and $\mathrm{Rh}$ ) in automotive exhaust control catalysis $[11,12]$ due to their high hydrothermal stability, high catalytic activity and low costs. Various La-containing oxides demonstrate a high dielectric loss. These materials have thus been considered as sensitising materials. One application is the used of such materials as a catalytic coating in the application of microwave-assisted in-situ soot filter regeneration for Diesel engines. Takatsu et al. [13] reported about such an application for $\mathrm{LaCoO}_{3}$ and demonstrated that this material can be rapidly heated by dielectric means and is thus applicable in cold-start applications for Diesel engines.

Dielectric properties of perovskites have been widely studied. Setter et al. [9] have reported that dielectric permittivities of perovskites are closely related to polarizability and to the dilution of dipoles caused by thermal expansion on A and B sites. Ivanov et al. [14] noticed that phase transition can cause a jump in dielectric permittivity for $\mathrm{La}_{1-x} \mathrm{Sr}_{x} \mathrm{MnO}_{3}$ at sub-millimeter wavelengths. Phase transitions found in complex perovskites are linked to ion packing densities. Therefore, the dielectric permittivity has been correlated to the tolerance factor, ionic size. It was found that dielectric loss is lower when the tolerance factor was closer to unity [8]. Shan et al. [15] have 
reported that a decreasing in dielectric constants with for higher atomic number of $\mathrm{Ln}$ (from Dy to $\mathrm{Lu}$ ) for $\mathrm{Ln}_{0.5} \mathrm{Na}_{0.5} \mathrm{TiO}_{3}$. Dwivedi et al. [16], reports that the dielectric constant of $\mathrm{Ca}_{1-x} \mathrm{La}_{x}\left(\mathrm{Ti}_{1-x} \mathrm{Cr}_{x}\right) \mathrm{O}_{3}$ first increase for $x=0.01-0.3$, and subsequently decreases for $x$ increasing up to 0.5 at room temperature.

In the present study, we investigate the dielectric properties of $\mathrm{LaMnO}_{3}$ in particular with regard to the influence of substitution of $\mathrm{La}$ in $\mathrm{LaMnO}_{3}$ by $\mathrm{Ce}$. Substitution of $\mathrm{Ce}$ has been shown to lead to a higher activity in oxidation of $\mathrm{CH}_{4}$ during dielectric heating [17], which explains its widespread use in automotive catalysis. The dielectric properties are further related to the bulk structure, mean crystallite size and surface morphology. Measurements were carried out on pressed perovskite pellets using an open-ended coaxial line dielectric probe method, and corrected for the density. Finally the thermal stability and sintering behaviour were probed.

\section{Theory}

\section{Dielectric permittivity}

The dielectric properties of a material can be characterised by the (frequency-dependent) values of the dimensionless relative dielectric permittivity $(\varepsilon)$, relative to the permittivity of vacuum $\left(\varepsilon_{0}=8.85 \times 10^{-12} \mathrm{~F} \mathrm{~m}^{-1}\right)$. For a real dielectric, $\varepsilon$ is a complex value $[18,19]$ :

$\varepsilon=\varepsilon^{\prime}-j \varepsilon^{\prime \prime}$

The real part $\varepsilon^{\prime}$ is the dielectric constant, which is a measure of the amount of energy that can be stored in a material in the form of electric field. The imaginary part $\varepsilon^{\prime \prime}$ is termed the loss factor, which is a direct measure of how much energy a material can dissipate in the form of heat. When a material is placed in a microwave field, the power absorbed by the material $P$ $\left(\mathrm{W} \mathrm{m}^{-3}\right)$ is a function of the applied microwave frequency $f(\mathrm{MHz})$, the electric field inside the material $E\left(\mathrm{kV} \mathrm{m}^{-1}\right)$, and the complex dielectric permittivity of the material:

$P=2 \cdot \pi \cdot f \cdot \varepsilon_{0} \cdot \varepsilon^{\prime \prime} \cdot E_{\mathrm{rms}}^{2}$.

Dielectric mixture equations for pellets

The permittivity is a volumetric property and depends on the density, which is strongly variable for powder materials. Various dielectric mixing formulas are known which theoretically take into account the shape and structure of the inclusions in the mixture, but they are limited applicable in practice. The empirical exponential model is most frequently encountered [20, 21]:

$\varepsilon_{\mathrm{m}}^{k}=\sum_{i} \Phi_{i} \varepsilon_{i}^{k}$

where $\varepsilon_{\mathrm{m}}$ is the permittivity of mixture and $\Phi_{i}$ the volume fraction of the component $i$. Nelson [22] found that for dry carbohydrate and coal powders it fits quite well for $k=1 / 3$. Since the similarity of the dielectrical properties of the perovskite powders with these materials, it seems to be legitimate to use this relation. For mixtures with air $(\varepsilon=1+0 i)$ the equations reduces to the Landau, Liftshitz and Looyenga relation [23]:

$\left(\varepsilon_{\mathrm{m}}\right)^{1 / 3}=\Phi \cdot\left(\varepsilon_{2}^{1 / 3}-1\right)+1 ; \Phi=\frac{\rho_{\mathrm{m}}}{\rho_{2}}$

with $\rho_{\mathrm{m}}$ the mixture density and $\rho_{2}$ the lattice density of the material. In order to calculate the real and imaginary parts of the dielectric permittivity $\varepsilon_{2}$ of the solid material, an analytical expression for both parts is needed. As the analytical complex separated solution is rather cumbersome, the following approximation is used, since the imaginary part is often smaller than the real part:

$(a+i \cdot b)^{k}=a^{k}+i \cdot \frac{a^{k}}{a} \cdot k \cdot b ; a \gg|b|$

By rewriting the Looyenga equation in a complex separated approximation, the following relations are found between $\varepsilon_{\mathrm{m}}\left(\varepsilon_{\mathrm{m}}=\varepsilon_{\mathrm{mr}}+\mathrm{i} \varepsilon_{\mathrm{mi}}\right)$ and $\varepsilon_{2}\left(\varepsilon_{2}=\varepsilon_{2 \mathrm{r}}+\mathrm{i} \varepsilon_{2 \mathrm{i}}\right)$ :

$\varepsilon_{\mathrm{mr}}=\left[\Phi \cdot \varepsilon_{2 \mathrm{r}}^{k}+1-\Phi\right]^{\frac{1}{k}}$

$\varepsilon_{\mathrm{mi}}=\left[\Phi \cdot \varepsilon_{2 \mathrm{r}}^{k}+1-\Phi\right]^{\frac{1-k}{k}} \cdot \varepsilon_{2 \mathrm{r}}^{k-1} \cdot \Phi \cdot \varepsilon_{2 \mathrm{i}}$.

\section{Experimental}

Materials preparation

A series of $\mathrm{La}_{1-x} \mathrm{Ce}_{x} \mathrm{MnO}_{3}$ perovskites with $x=0,0.05$, $0.1,0.2$ and 0.3 was prepared by co-precipitation of $0.5 \mathrm{~N}$ aqueous solutions of the corresponding nitrates with $0.5 \mathrm{~N} \mathrm{NaOH}$ and $\mathrm{H}_{2} \mathrm{O}_{2}\left(\mathrm{Mn}: \mathrm{H}_{2} \mathrm{O}_{2}=1.8\right)$ at $323 \mathrm{~K}$ and a $\mathrm{pH}$ of around 9.1. Subsequently, the obtained precipitate was filtered off, washed, dried overnight at $393 \mathrm{~K}$ in air, and calcined in air at $1,023 \mathrm{~K}$ for $6 \mathrm{~h}$. 
Further details have been described elsewhere [24, 25]. For comparison, a commercial $\mathrm{La}_{0.8} \mathrm{Ce}_{0.2} \mathrm{MnO}_{3}$ perovskite $(99 \%)$ prepared by combustion spray pyrolysis was obtained from Praxair Specialty Ceramics.

\section{Structural characterisation}

The bulk structure of the perovskites before and after dielectric heating in helium was determined by a CPS 120 (XRD) equipped with a primary monochromator using $\mathrm{CuK} \alpha$ radiation, with $2 \theta$ in the range from $5^{\circ}$ to $125^{\circ}$. Solid densities are measured by a Multivolume Pycnometer 1305 using $\mathrm{He}$ as filling gas. The surface areas before and after dielectric heating were determined by $\mathrm{N}_{2}$-physisoption at $77 \mathrm{~K}$ on a Sorptomatic 1990 (CE Instruments) and evaluated using the BET equation. The surface morphology of the perovskites was studied by using a Jeol High resolution SEM (JSM6000F) with a Field Emission Gun FEG.

Dielectric heating experiments

\section{Experimental set-up}

Dielectric heating was carried out in a microwave setup consisting of three parts: inlet manifolds, a microwave system with a reactor and a Mass Spectrometry gas composition analyser (Prisma QMS 2000) (Fig. 1). The microwave system is operated at $2.45 \mathrm{GHz}$ in a travelling wave once-through system with continuously adjustable power (Muegge, maximum $1 \mathrm{~kW}$ ). This system consists of a three-stub tuner section (Muegge), a monomode microwave cavity $\mathrm{TE}_{10}$, a circulator (Philips) connected to a water load and a water dummy load located at the end of the copper waveguide. The stub tuners protect the microwave source against reflected radiation. The incident reflected and transmitted power is measured by a power meter (Rhode \& Schwarz). A quartz sample holder (i.d. $=18 \mathrm{~mm}$ ) is placed perpendicularly to the direction of propagation in order to achieve maximum microwave absorption by the tested samples. For all experiments, the bed volume of the sample was kept constant $\left(10 \mathrm{~cm}^{3}\right)$.

The temperature in the sample bed is controlled by implementing a control loop over the microwave power. The temperature of the sample bed is assessed by an optical fibre (Luxtron, Accufiber-OFT straight end lightpipe) with a lower detection limit of $373 \mathrm{~K}$. Calibration of the optical fibre was carried out by heating both the fibre and a thermocouple conventionally between $473 \mathrm{~K}$ and $923 \mathrm{~K}$.

\section{Dielectric heating procedure}

Prior to dielectric heating, the as-prepared sample powders were placed in the quartz reactor and dried in a conventional oven to $423 \mathrm{~K}$ in a He flow (gas hourly space velocity, GHSV $=600 \mathrm{~h}^{-1}$ ) with a
Fig. 1 Schematic drawing of the microwave set-up operated at $2.45 \mathrm{GHz}$ in travelling wave once-though mode with a microwave source with continuously adaptable power (max. $1 \mathrm{~kW})$

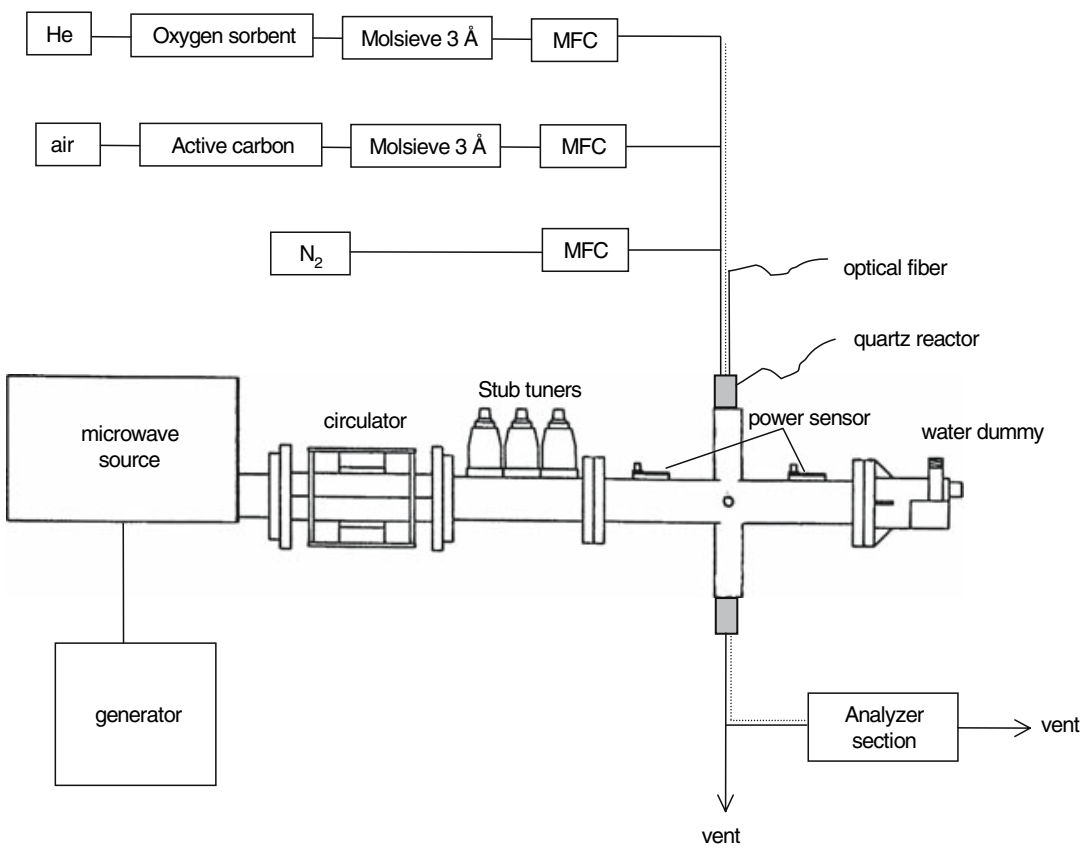


heating rate of $5 \mathrm{~K} \mathrm{~min}^{-1}$. After cooling down in $\mathrm{He}$ to room temperature, the reactor containing the powder was transported to the microwave cavity. Dielectric heating was carried out to $873 \mathrm{~K}$ in $\mathrm{He}$ $\left(\mathrm{GHSV}=600 \mathrm{~h}^{-1}\right)$ at $2 \mathrm{~K} \mathrm{~min}^{-1}$. The sample was subsequently kept at that temperature for $24 \mathrm{~h}$. Following this the samples (still in the He flow) were rapidly heated at an incident power of $200 \mathrm{~W}$ for $1 \mathrm{~h}$ and cooled down to room temperature. This was repeated five times in order to test the thermal stability during dielectric heating.

\section{Dielectric permittivity measurements}

The dielectric permittivities of the perovskites near the microwave frequency (from $30 \mathrm{MHz}$ to $3 \mathrm{GHz}$ ) were obtained at room temperature by an open-ended coaxial line dielectric probe [26]. A network analyser (HP 8752A) constitutes both the source and the detector of electromagnetic waves for a range of defined frequencies. The analyser is coupled by a coaxial line to an open-ended probe (Agilent HP $85070 \mathrm{C}$ ), which has to be in direct contact with the sample to be characterised. A schematic view is shown in Fig. 2. The end of the coaxial line, defined by the probe itself and the sample, represents a capacity composed of the internal probe capacity and the fringing field capacity, which is determined by the permittivity of the sample. This capacity influences the phase and amplitude of the reflected signal back to the network analyser. For good results, it is required that the samples are homogeneous and have flat surfaces. To this end, the perovskites were pressed to pellets with $10 \mathrm{~mm}$ diameter by $4 \mathrm{~mm}$ high by using a Benchtop Single Punch Tablet Press Set up (TDP model) at a maximum pressure of 1.5 ton. Pellet densities were assessed over their weight and volume.

\section{Results}

Bulk structure and surface morphology of fresh La-Ce-Mn perovskites

After calcination at $1,073 \mathrm{~K}$, well-defined perovskite crystalline structures are obtained for perovskites with $x=0,0.05$ and 0.1 (Fig. 3). For higher degrees of $\mathrm{Ce}$ substitution, cerium is not entirely incorporated into the perovskite lattice, and two characteristic $\mathrm{CeO}_{2}$ peaks with reflections at $3.14 \AA$ and $1.63 \AA$ can be observed for $x=0.2$ and 0.3. A small shift towards smaller $d$-values in the perovskite lattice constants is observed for increasing $\mathrm{Ce}$ contents. The average crystallite sizes was assessed using the Scherrer equation for reflections at $2 \theta=22.8^{\circ}$ and $28.3^{\circ}$ for perovskite and $\mathrm{CeO}_{2}$, respectively (Table 1). Substitution of La by Ce clearly results in a decrease of the crystallite size. Also the preparation method is relevant: for the commercial $\mathrm{La}_{0.8} \mathrm{Ce}_{0.2} \mathrm{MnO}_{3}$ perovskite prepared by combustion spray pyrolysis, much larger crystallite sizes are found for both the perovskite and the $\mathrm{CeO}_{2}$ phases than for the co-precipitated sample.

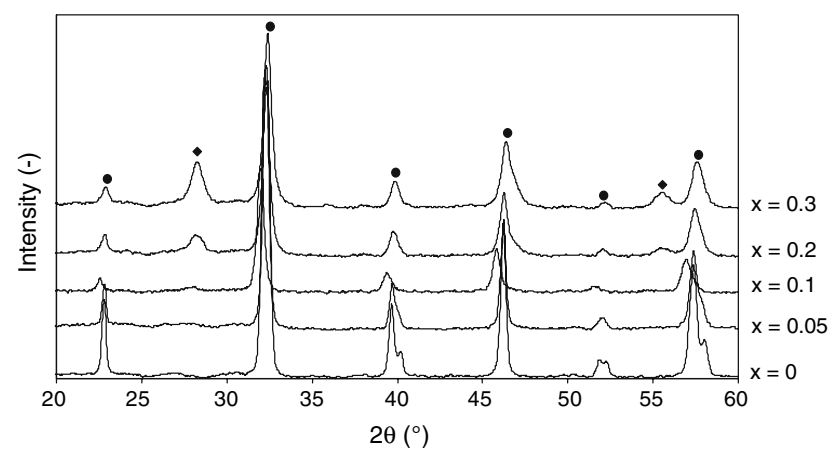

Fig. 3 XRD patterns of $\mathrm{La}_{1-x} \mathrm{Ce}_{x} \mathrm{MnO}_{3}$ powder $(x=0,0.05,0.2$ and 0.3$)$ calcined at $1,073 \mathrm{~K}$ in air for $6 \mathrm{~h}$. (•): perovskite reflections; $(\bullet) \mathrm{CeO}_{2}$ reflections
Fig. 2 Schematic drawing of measuring system for dielectric permittivity: open-ended coaxial line dielectric probe; E: electric field

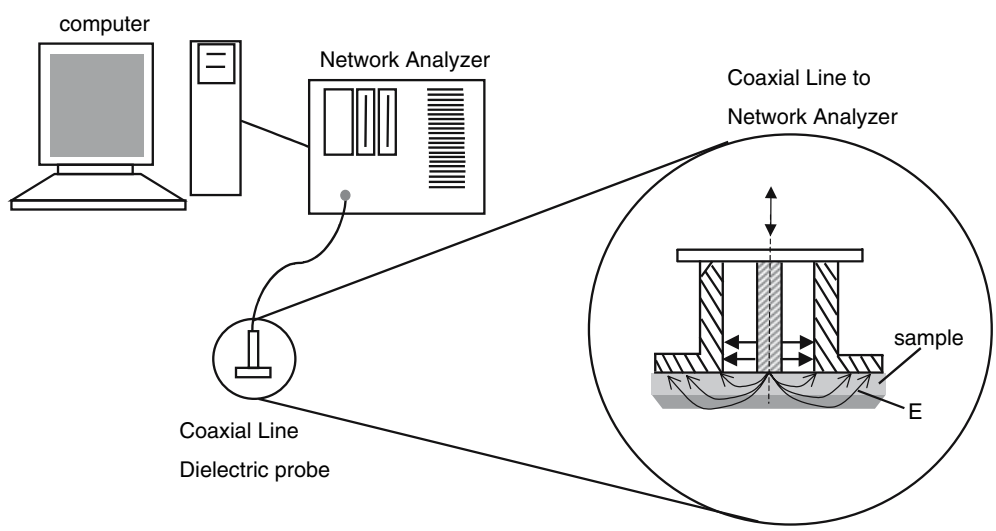


Table 1 Average crystallite sizes of $\mathrm{La}_{1-x} \mathrm{Ce}_{x} \mathrm{MnO}_{3}$ perovskites with $x=0,0.05,0.1,0.2$ and 0.3 before and after dielectric heating, estimated by means of the Scherrer equation

\begin{tabular}{llllll}
\hline$x$ & $\begin{array}{l}\text { Average crystallite size } \\
\text { (fresh) }(\mathrm{nm})\end{array}$ & & \multicolumn{2}{l}{$\begin{array}{l}\text { Average crystallite size } \\
(\mathrm{nm})(\mathrm{after} \text { dielectric } \\
\text { heating) }\end{array}$} \\
\cline { 2 - 3 } & Perovskite & $\mathrm{CeO}_{2}$ & & Perovskite & $\mathrm{CeO}_{2}$ \\
\hline 0 & 26.1 & n.a. & & 13.6 & n.a. \\
0.05 & 23.7 & n.a. & & 10.6 & n.a. \\
0.1 & 20.1 & n.a. & & n.a. & n.a. \\
0.2 & 15.4 & 9.5 & & 12.0 & 12.1 \\
$0.2^{\mathrm{a}}$ & 23.9 & 17.0 & & n.a. & n.a. \\
0.3 & 13.1 & 11.0 & 13.0 & 13.8 \\
\hline
\end{tabular}

${ }^{a}$ Combustion spray pyrolysis

n.a.: not applicable

A high degree of cerium substitution results in microstructural changes, which can be observed by SEM (Fig. 4). The surface image of $\mathrm{LaMnO}_{3}$ demonstrates that this material consists of uniform particles with sizes of about $0.1 \mu \mathrm{m}$, whereas the morphology of $\mathrm{La}_{0.8} \mathrm{Ce}_{0.2} \mathrm{MnO}_{3}$ has a layered and agglomerated structure.

\section{Dielectric heating}

All perovskites are sensitive to microwave irradiation and can easily be heated. $\mathrm{La}_{0.8} \mathrm{Ce}_{0.2} \mathrm{MnO}_{3}$ demonstrates a high thermal stability. The dielectric loss factor and thus the final temperature obtained remain constant over a $24 \mathrm{~h}$ period (figure not shown). Moreover, the perovskites are stable with respect to rapid and repeated heating, as illustrated for $\mathrm{LaMnO}_{3}$ in Fig. 5 for repeated heating/cooling cycles. A constant temperature of $750 \mathrm{~K}$ was reached during these cycles.

The fraction of absorbed microwave increases with temperature (Fig. 6a). For $x=0$ and 0.05 , a sudden

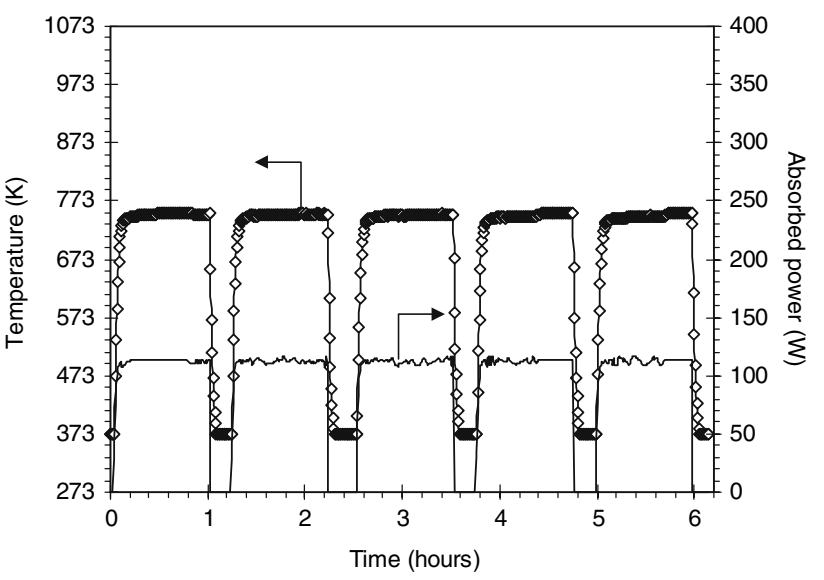

Fig. 5 Temperature response and absorbed power for five subsequent dielectric heating cycles under a $\mathrm{He}$ flow $\left(\mathrm{GHSV}=600 \mathrm{~h}^{-1}\right)$ at an incident power of $200 \mathrm{~W}$ for $\mathrm{LaMnO}_{3}$ powder

increase in power absorbed is observed at $450 \mathrm{~K}$ and $440 \mathrm{~K}$, respectively, which is accompanied by a small release of oxygen. No direct relation can be observed between the amount of $\mathrm{Ce}$ substitution and the absorbed microwave power. However, a linear relation is found between the bulk density and the absorbed microwave power (Fig. 6b), although the value for $x=0.3$ is somewhat low with respect to the other values.

No significant change in bulk structure is observed after the dielectric heating (Fig. 7), although for $\mathrm{La}_{0.7} \mathrm{Ce}_{0.3} \mathrm{MnO}_{3}$ two peaks appear at $46.7^{\circ}$ and $47.5^{\circ}$ replacing a single peak at $47.1^{\circ}$. Mean crystallite sizes and specific surface areas of the tested perovskites after heating are given in Table 1 and 2, respectively. For all tested perovskites, some sintering occurs as evidenced by a loss in surface area is observed after heating in helium.
Fig. 4 Surface morphology of $\mathrm{LaMnO}_{3}$ powder (a) and $\mathrm{La}_{0.8} \mathrm{Ce}_{0.2} \mathrm{MnO}_{3}$ powder (b) obtained by SEM
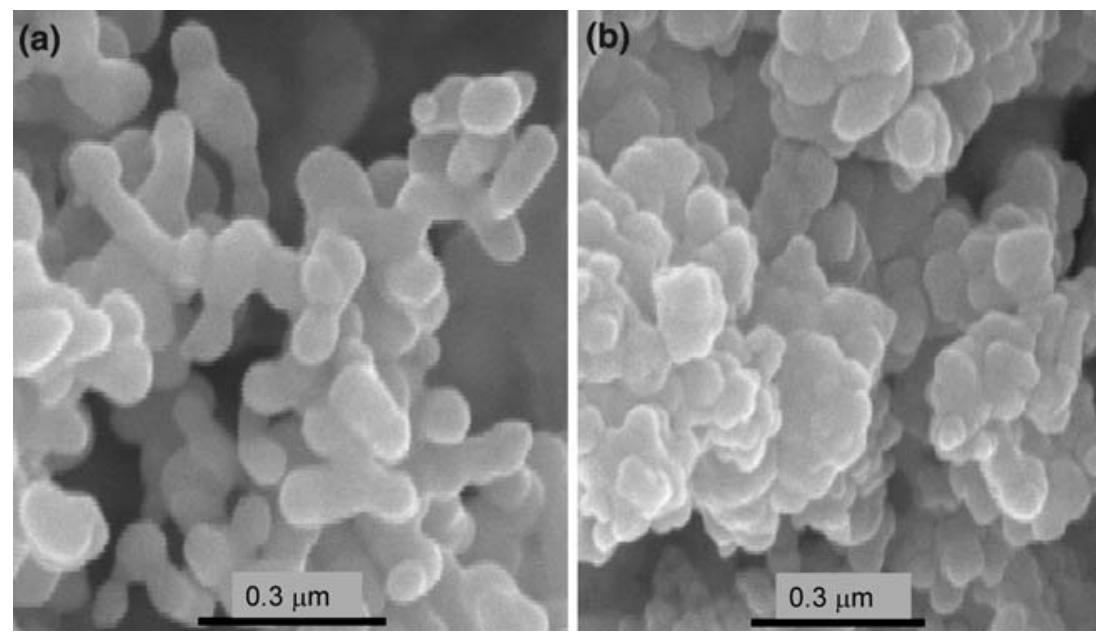

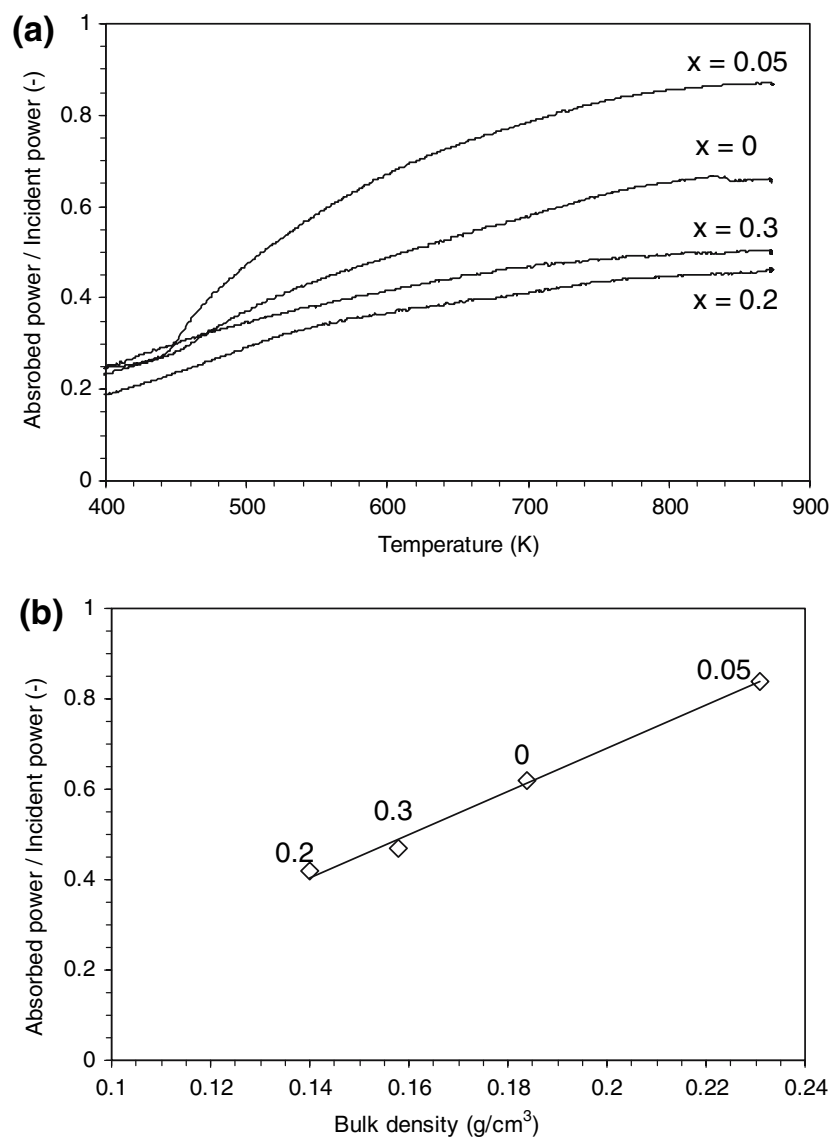

Fig. 6 Absorbed power as a function of temperature (a) and bulk density verses the absorbed power (b) during dielectric heating $\left(2 \mathrm{~K} \mathrm{~min}^{-1}\right)$ in helium $\left(\mathrm{GHSV}=600 \mathrm{~h}^{-1}\right)$ for $\mathrm{La}_{1-x} \mathrm{Ce}_{x} \mathrm{MnO}_{3}$ perovskite powders with $x=0,0.05,0.2,0.3$

\section{Dielectric permittivity}

The dielectric permittivity of solid perovskites, as calculated according to Eqs. 6-7, are represented in
Table 2 Specific surface areas of $\mathrm{La}_{1-x} \mathrm{Ce}_{x} \mathrm{MnO}_{3}$ perovskite powders with $x=0,0.05,0.1,0.2$ and 0.3 before and dielectric heating. Heating procedure: heating to $873 \mathrm{~K}$ at $2 \mathrm{~K} \mathrm{~min}^{-1}$ in $\mathrm{He}$ $\left(\mathrm{GHSV}=600 \mathrm{~h}^{-1}\right.$ ) for $24 \mathrm{~h}$, subsequent cooling down to room temperature in helium, followed by rapid and repeated heating at an incident power of $200 \mathrm{~W}$ for five times

\begin{tabular}{lll}
\hline Sample & $\begin{array}{l}\text { Surface area before } \\
\text { heating }\left(\mathrm{m}^{2} \mathrm{~g}^{-1}\right)\end{array}$ & $\begin{array}{l}\text { Surface area after } \\
\text { heating }\left(\mathrm{m}^{2} \mathrm{~g}^{-1}\right)\end{array}$ \\
\hline $\mathrm{LaMnO}_{3}$ & 10.0 & 2.3 \\
$\mathrm{La}_{0.95} \mathrm{Ce}_{0.05} \mathrm{MnO}_{3}$ & 17.1 & 5.3 \\
$\mathrm{La}_{0.9} \mathrm{Ce}_{0.1} \mathrm{MnO}_{3}$ & 26.1 & n.a. \\
$\mathrm{La}_{0.8} \mathrm{Ce}_{0.2} \mathrm{MnO}_{3}$ & 41.0 & 21 \\
$\mathrm{La}_{0.7} \mathrm{Ce}_{0.3} \mathrm{MnO}_{3}$ & 26.8 & 6.5 \\
\hline
\end{tabular}

${ }^{a}$ Not available

Fig. 8. For all tested samples, both the dielectric constant and the loss factor decrease at higher frequencies. However, for $x=0.05$, the loss factor slightly increases at higher frequencies. Both the measured dielectric properties of the perovskite tablets and those calculated for the solid material at $2.45 \mathrm{GHz}$ are summarised in Table 3.

\section{Discussion}

Crystalline structure related to Ce substitution

The small shift in the perovskite lattice constants for a higher degree of substitution of $\mathrm{La}$ by $\mathrm{Ce}$ is expected as $\mathrm{Ce}$ has a somewhat smaller ion radius than La. The disappearance of a well-defined crystalline structure with larger amounts of $\mathrm{Ce}$ is indicated by the smaller crystallite sizes. A larger volume of a disordered (amorphous) structure is formed in the grain boundaries. This can be explained by strain induced by Ce substitution, either
Fig. 7 XRD patterns of $\mathrm{La}_{1-x}$ $\mathrm{Ce}_{x} \mathrm{MnO}_{3}$ perovskite powders with $x=0,0.05,0.2$ and 0.3 before and after microwave heating; $(\bullet)$ perovskite reflections; $(\bullet) \mathrm{CeO}_{2}$ reflections
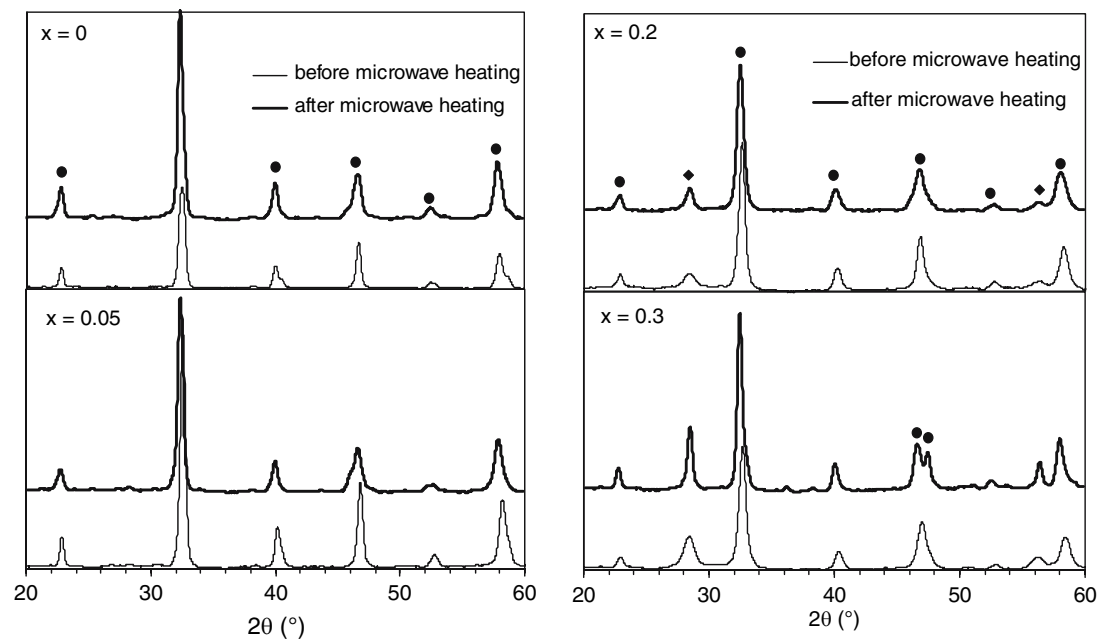

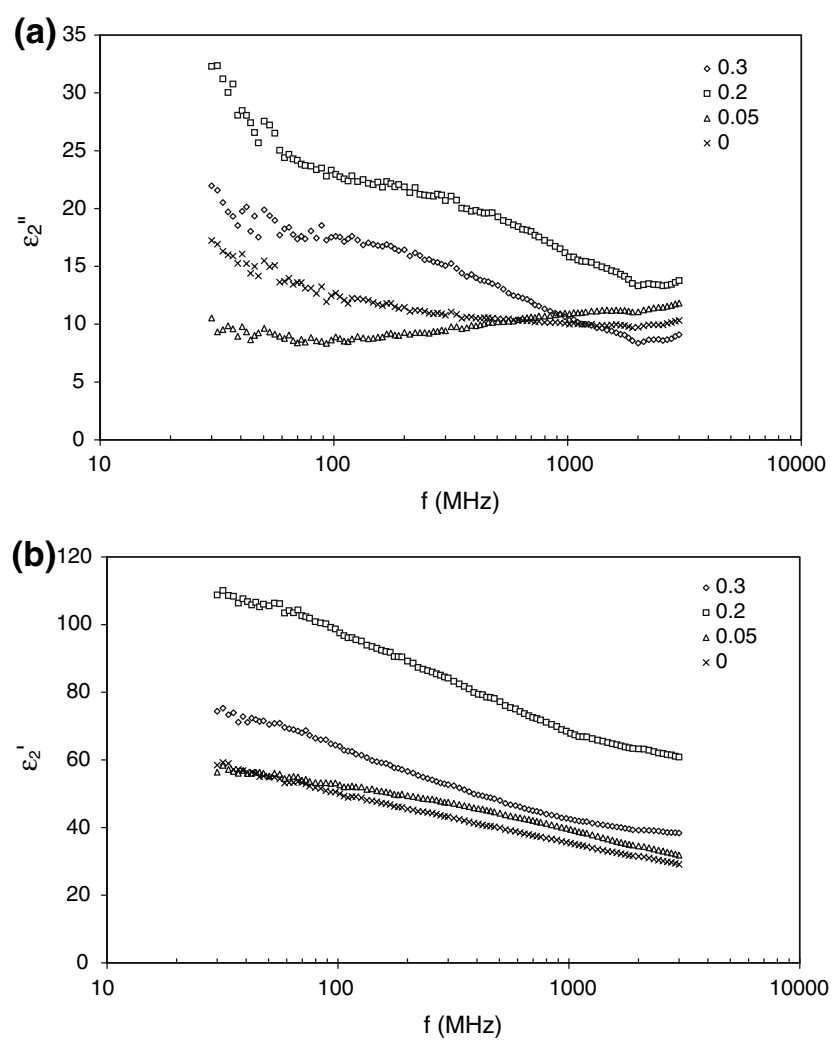

Fig. 8 Dielectric loss factor $\left(\varepsilon_{2}{ }^{\prime \prime}\right)$ (a) and dielectric constant of solid materials $\left(\varepsilon_{2}^{\prime}\right)(\mathbf{b})$ as a function of frequency at room temperature for $\mathrm{La}_{1-x} \mathrm{Ce}_{x} \mathrm{MnO}_{3}$ pellets, with $x=0,0.05,0.2$ and 0.3 , as calculated from generalised Looyenga equation

due to size mismatch of $\mathrm{Ce}$ or to the creation of oxygen defects, and is in line with the formation of a less-ordered surface morphology, as shown by SEM. The crystallite size of the $\mathrm{CeO}_{2}$ phase increases slightly with $\mathrm{Ce}$ substitution, indicating the formation of well-defined crystalline $\mathrm{CeO}_{2}$.

Dielectric behaviour during heating

All tested perovskites are capable to absorb microwave energy. The absorbed microwave power increases at

Table 3 Tablet and solid densities, measured $\varepsilon_{\mathrm{m}}{ }^{\prime}$ and $\varepsilon_{\mathrm{m}}{ }^{\prime \prime}$, solid dielectric constant $\varepsilon_{2}^{\prime}$ and loss factor $\varepsilon_{2}^{\prime \prime}$ as calculated from Eqs. $6-7$, and loss factor $\varepsilon_{2}^{\prime \prime}$ normalised with respect to lattice density, higher temperature, indicating an increase of the dielectric loss factor [19]. This is to be expected since the loss is composed of both conduction and dipolar reorientation losses. With temperature, the conduction increases as does the frequency of maximum dipolar loss. The jump in absorbed power at low temperatures for $x=0$ and 0.05 may be related to desorption of weakly bonded surface oxygen, as some oxygen was detected by MS. This may lead to a sudden increase in bulk density (sintering), which explains the increase in absorbed power. No gas release was detected for $x=0.2$ and 0.3 , which can be explained by the oxygen deficient structure created by Ce substitution [24]. The linear correlation between the absorbed power and the bulk density of the powders is an indication that the loss factor is density dependent. This clearly indicates that the final sintering density is the prominent parameter that rules the microwave absorption of the perovskite samples. The change in microwave absorption is not caused by a sudden increase in the (densitycorrected) loss factor, which is known to give rise to run-away effects during dielectric heating.

The long-term heating and rapid and temperature cycling experiments suggest a high thermal stability of the perovskite structure. This is confirmed by XRD, showing that the perovskite retains a well-defined crystal structure after heating. However, the BET surface areas of the powders decreased dramatically. The least sintering was observed for $\mathrm{La}_{0.8} \mathrm{Ce}_{0.2} \mathrm{MnO}_{3}$. The perovskite crystallites did not grow during heating, while a small increase of the $\mathrm{CeO}_{2}$ crystallite size was found, suggesting some sintering only of the $\mathrm{CeO}_{2}$ phase.

\section{Dielectric properties}

The dielectric loss factor $\left(\varepsilon_{\mathrm{m}}{ }^{\prime \prime}\right)$ of the perovskites at $2.45 \mathrm{GHz}$ at room temperature is about 3 , which is roughly of the same order as the value of soot [19]. A decrease in dielectric loss with higher frequencies is

at $2.45 \mathrm{GHz}$ and at $298 \mathrm{~K}$ for $\mathrm{La}_{1-x} \mathrm{Ce}_{x} \mathrm{MnO}_{3}$ perovskite pellets $(x=0,0.05,0.2,0.3)$

\begin{tabular}{|c|c|c|c|c|c|c|c|}
\hline \multirow[t]{2}{*}{$x$} & \multicolumn{2}{|c|}{ Density $\left(\mathrm{g} \mathrm{cm}^{-3}\right)$} & \multicolumn{2}{|c|}{ Permittivity tablet } & \multicolumn{3}{|c|}{ Solid perovskites } \\
\hline & Tablet $(\rho)$ & Solid $\left(\rho_{2}\right)$ & $\varepsilon_{\mathrm{m}}^{\prime}$ & $\varepsilon_{\mathrm{m}}^{\prime \prime}$ & $\varepsilon_{2}^{\prime}$ & $\varepsilon_{2}^{\prime \prime}$ & $\varepsilon_{2}^{\prime \prime} /\left(\rho_{2}\right)$ \\
\hline 0 & 3.53 & 4.26 & 20.90 & 6.74 & 30.34 & 9.95 & 2.34 \\
\hline 0.05 & 3.73 & 4.65 & 21.17 & 6.74 & 33.33 & 11.46 & 2.46 \\
\hline 0.2 & 2.56 & 4.13 & 23.08 & 4.33 & 62.07 & 13.41 & 3.25 \\
\hline $0.2^{\mathrm{a}}$ & 3.90 & 4.69 & 21.02 & 3.60 & 30.53 & 5.58 & 1.19 \\
\hline 0.3 & 2.60 & 3.95 & 17.55 & 3.41 & 38.98 & 8.69 & 2.20 \\
\hline
\end{tabular}

${ }^{\mathrm{a}} \mathrm{La}_{0.8} \mathrm{Ce}_{0.2} \mathrm{MnO}_{3}$ perovskite prepared by combustion spray pyrolysis 
observed for all tested samples except for $\mathrm{La}_{0.95}$ $\mathrm{Ce}_{0.05} \mathrm{MnO}_{3}$. At the lower frequencies the dominant mechanism of energy transfer to a dielectric material is through conductive currents flowing due to the movement of ionic constituents. The effect of this mechanism can be enhanced by the presence of impurities, and decreases proportional to the frequency. At higher microwave frequencies (around $1 \mathrm{GHz}$ ), energy is primarily absorbed by reorientation of permanent dipole molecules. For $x=0,0.2$ and 0.3 , the inverse relation of $\varepsilon_{2}$ " with frequency in the low- $f$ region thus indicates a strong effect of ionic constituents along with the existence of weak molecular dipole moments. The remarkable slight increase in dielectric loss with increasing frequency for $x=0.05$ may be explained by only a very weak loss by conduction through ions, with loss through dipole reorientation being the dominant mechanism. The minor contribution of ionic conduction could be related to Ce doping, minimising the number of ionic dipoles (possibly oxygen vacancies or interstitials).

As the dielectric loss is determined by the number of dipoles, this value is expected to be density-dependent. For this reason, the values have been given after division through the lattice density $\rho_{2}$ (Fig. 9). At frequencies above $1 \mathrm{GHz}$, an increase in the densitycorrected dielectric loss with rising Ce substitution is found, with a somewhat lower value for $x=0.3$.

The gradual decrease of the dielectric constant with rising frequency for all tested samples is also representative of the loss of coupling of ionic sites to the field at higher frequencies. Here, again, the sample with $x=0.05$ is deviant from the others, with a much smaller slope at low frequencies. This can likewise be explained by a smaller number of ionic dipoles in this material.

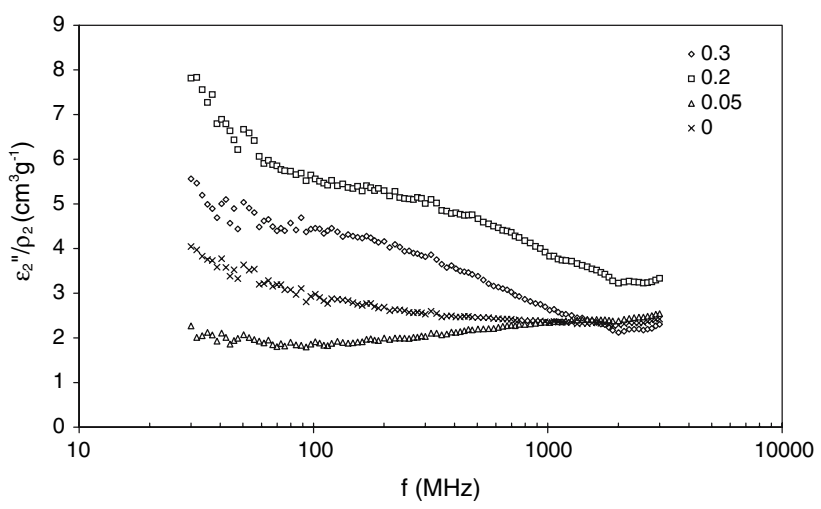

Fig. 9 Density corrected loss factor $\left(\varepsilon_{2}^{\prime \prime} / \rho 2\right)$ as function of frequency at room temperature for $\mathrm{La}_{1-x} \mathrm{Ce}_{x} \mathrm{MnO}_{3}$ pellets, $x=0$, $0.05,0.2$ and 0.3
The measured dielectric constants of the pellets and the calculated value of the solid materials at $2.45 \mathrm{GHz}$ show an increase for cerium substitution up to $x=0.2$, followed by a smaller value for $x=0.3$. A similar trend is found for the density-corrected dielectric loss of solid perovskites. The smaller values at $x=0.3$ can well be explained by the presence of a separate $\mathrm{CeO}_{2}$ phase with a low dielectric constant (2.8) [27] and low dielectric loss [28], which results in a net drop in both $\varepsilon^{\prime}$ and $\varepsilon^{\prime \prime}$.

In the low- $f$ region, the dielectric constants and loss factors up to $x=0.2$ correspond to a drop in crystallite size and bulk density. Small crystallite sizes and low bulk densities can be explained by lattice strain and by a higher concentration of cation/oxygen vacancies. The latter could lead to a higher concentration of ionic dipoles and thus to higher values for $\varepsilon^{\prime}$. The presence of vacancies has also been confirmed in earlier work [24], and related to oxidation activity. Clearly, the dielectrical properties of this material are a good indication of the activity-related number of defects. For $x=0.05$, it may be suggested that the total number of oxygen and cation vacancies has become minimised due to $\mathrm{Ce}$ substitution.

The dielectric constants and loss factors are influenced by the bulk composition but also by the method of preparation. The materials prepared by co-precipitation and by combustion spay pyrolysis show different results, suggesting a less defective structure for the latter material.

\section{Conclusion}

Interaction with microwave irradiation has been studied for $\mathrm{LaMnO}_{3}$ perovskites with a varying degree of Ce doping. High dielectric constants $\varepsilon^{\prime}$ have been found, as well as intermediate dielectric losses $\varepsilon^{\prime \prime}$. The $\varepsilon^{\prime \prime}$ values slightly increase with temperature, but do not give rise to runaway effects, making the materials highly suitable for dielectric heating. While some sintering occurs during dielectric heating in helium, no significant changes in the bulk structure were observed after dielectric heating, indicating sufficient thermal stability for all perovskites. The highest thermal stability was found for $\mathrm{La}_{0.8} \mathrm{Ce}_{0.2} \mathrm{MnO}_{3}$. For $x=0.05$, a comparatively small dielectric constant and loss factor are observed at low frequencies, indicating a small contribution of ionic conduction. This may be attributed to effective annihilation of ionic defects for this amount of $\mathrm{Ce}$ doping. At microwave frequencies, dipolar reorientation mechanisms are dominant. An increase of the dielectric constant and dielectric loss is 
found with increasing amount of $\mathrm{Ce}$ up to $x=0.2$, which can be attributed to a larger number of cation/ oxygen vacancies. For $x=0.3$, both the dielectric loss and dielectric constant decrease, as a result of the formation of a separate $\mathrm{CeO}_{2}$ phase with low values for $\varepsilon^{\prime}$ and $\varepsilon^{\prime \prime}$.

Acknowledgements Financial support of the Netherlands Research Council (STW) is gratefully acknowledged. Thanks are due to Mr. P. F. Collignon, H. Agema and J. Beckers for their technical support.

\section{References}

1. Huang CL, Chen YC (2002) Jpn J Appl Phys 41:1459

2. Chen HL, Huang CL (2002) Jpn J Appl Phys 41:5650

3. Cho JY, Yoon KH, Kim ES (2002) Jpn J Appl Phys 41:4601

4. Kim WS, Yoon KH, Kim ES (2000) Jpn J Appl Phys 39:5650

5. Yoon KH, Chang YH, Kim WS, Kim JB, Kim ES (1996) Jpn J Appl Phys 35:5145

6. Kagata H, Kato J (1994) Jpn J Appl Phys 33:5463

7. Colla EL, Reaney IM, Setter N (1993) J Appl Phys 74:3414

8. Zurmühlen R, Colla E, Dube DC, Petzelt J, Reaney I, Bell A, Setter N (1994) J Appl Phys 76:5864

9. Setter N, Colla E, Reaney I, Zurmühlen R, Dube D, Petzelt J (1994) Ferroelectrics 154:231

10. Peña MA, Fierro JLG (2001) Chem Rev 101:981

11. Libby WF (1972) Science 171:499

12. Voorhoeve RJH, Johnson DW Jr, Remeika JP, Gallagher PK (1977) Science 195:827
13. Takatsu K, Kurogi F, Matsue A, Kasaya M (1996) SAE paper 960344, p 69

14. Ivanov VY, Travkin VD, Mukhin AA, Lebedev SP, Volkov AA, Pimenov A, Loidl A, Balbashov AM, Mozhaev AV (1998) J Appl Phys 83:7180

15. Shan YJ, Nakamura T, Inaguma Y, Itoh M (1998) Solid State Ionics 108:123

16. Dwivedi RK, Kumar D, Parkash O (2001) J Mater Sci 36:3641

17. Zhang-Steenwinkel Y, Castricum HL, Beckers J, Eiser E, Bliek A (2004) J Catal 221:523

18. Metaxas AC, Meredith RJ (1983) Industrial microwave heating. Peter Peregrinus Ltd., London

19. Ma JX, Fang M, Li P, Zhu B, Lu XH, Lau NT (1997) Appl Catal A: Gen 159:211

20. Nelson SO, You TS (1990) J Phys D: Appl Phys 23:346

21. Nelson S, Kraszewski A, You T (1991) J Microwave Power Electromagnet Energy 26:45

22. Nelson SO (1983) Trans ASAE 26:1823

23. Roussy G, Pearcs JA (1995) In: Foundations and industrial applications of microwaves and radio frequency fields. John Wiley \& Sons, Chichester

24. Zhang-Steenwinkel Y, Beckers J, Bliek A (2002) Appl Catal A: Gen 235:79

25. Hackenberger M, Stephan K, Kie $\beta$ ling D, Schmitz W, Wendt G (1997) Solid State Ionics 101-103:1195

26. Regier M, Schubert H (2001) In: Thermal technologies in food processing. Woodhead Publishing Ltd., Cambridge

27. Hartmanova M, Gmucova K, Thurzo I (2000) Solid State Ionics 130:105

28. Michael D, Mingos P, Baghurst DR (1991) Chem Soc Rev 20:1 